\title{
A Sustematic Review of Skeletal, Dental and Soft Tissue Treatment Effects of Twin Block Appliance.
}

\author{
Dr Suchita Daokar,' Dr Mitali Sharma² \\ 'Professor, PG \& PhD Guide, ${ }^{2}$ PG Resident \\ Department of Orthodontics, CSMSS Dental College, Maharashtra, India \\ Correspondence: Dr Suchita Tarvade (Daokar); Email: suchitadaokar@gmail.com
}

\section{ABSTRACT}

This systematic review aims to assess skeletal, dental and soft tissue treatment effects of the conventional Twin-block appliance compared to control in Class II malocclusion till date. The electronic databases of Medline, PubMed and Cochrane library were systematically searched until September 2019. The human studies that had used the conventional Twin block appliance with a control group evaluated by a Lateral Cephalogram were included. A total of 321 articles were examined. 10 articles were selected. Majority studies reported a significant improvement in the mandible and in the vertical height, maxillary restrictive effect was less consistently reported. A restrictive maxillary molar effect and an advancement in the mandibular molar has been suggested. Retroclination of the maxillary incisors and proclination of mandibular incisor inclination has been reported. There is some evidence to suggest a retrusive upper lip effect and a protrusive lower lip effect. Increase in Nasolabial angle and some decrease in the mentolabial sulcus to be expected. In conclusion the conventional Twin Block appliance have shown desirable skeletal, dental and soft tissue effects. However certain inevitable side effects are also seen mainly its effect on lower anterior proclination.

Keywords: Systematic review, Twin block appliance, Treatment effects.

\section{INTRODUCTION}

The Twin block was developed by Dr William J. Clark in 1977.' Even though it's a removable appliance requiring patient compliance, it's still one of the most widely used functional correctors.

Twin-block appliance consists of upper and lower acrylic bite blocks with interlocking occlusal inclined planes at $70^{\circ}$ angle, which functions to bring the mandible in its desired forward and downward position. ${ }^{2}$ It's mainly indicated in actively growing Class II division I malocclusions. ${ }^{1,3}$

Multiple authors have reported variable results with variable intensities till-date. ${ }^{4-5}$ A recent systematic review reporting all the treatment effects of Twin Block was lacking.

The aim of this review is to assess the possible skeletal, dental and soft tissue treatment effects of the conventional Twin-block appliance compared to controls in Class II malocclusion individuals till date.

\section{MATERIALS AND METHODS}

\section{Information sources}

The electronic databases of Medline, PubMed and Cochrane library were systematically searched until September 2019. A limited grey-literature search was also done in Google Scholar.

\section{Search strategy}

Table 1 shows the terms used to carry out the search. Duplicate results were eliminated.

Inclusion criteria:

1. Human Cephalometric studies.

2. Use of conventional Twin-block appliance.

3. Cases treated with Non-extraction and Nonsurgical approach to prevent introduction of any confounding factors.

4. Comparison with control group of untreated Class II malocclusion cases. ${ }^{5}$ 
Table 1: Search data and search strategies.

\section{Database}

Medline/PubMed: 1989 to present

Cochrane library: 1989 to present

Google Scholar: 1989 to present

\section{Search strategy}

(twin block OR twin-block OR twinblock) AND [(treatment outcome OR treatment effect\$) OR (skeletal effect OR skeletal change) OR (dental effect OR dental change) OR (facial change or profile change or soft-tissue change)]
Number of Results

251

70

14,800

\section{Study selection}

For each database search, first the titles and abstracts were evaluated. The articles that did not match the inclusion criteria, case reports, literature reviews, systematic reviews, editorial articles were all excluded. Articles including animal studies, modified twin block, non-cephalometric analysis were excluded.
Selected abstracts were further subjected to scrutiny of the complete text. Also, when the abstracts were found to be unclear, the full text was obtained.

The 10 articles (FLOW CHART 1) which met all the inclusion criteria were finally included in the systematic review. Google Scholar did not reveal any different results than those from Pubmed/ Medline or Cochrane library. (FLOW CHART 2).

Electronic search - 321

(Medline/PubMed, Cochrane library)

From 321 Articles excluded by title -210

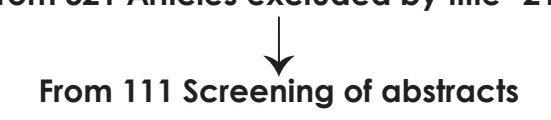

Exclusion of non-relevant studies- 81

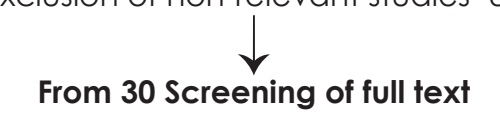

Exclusion of non-relevant studies- 20

Exclusion of non-relevant studies- 20

Study selection complete (10)

(10 studies meeting all criteria selected)

\section{Flow Chart 1}
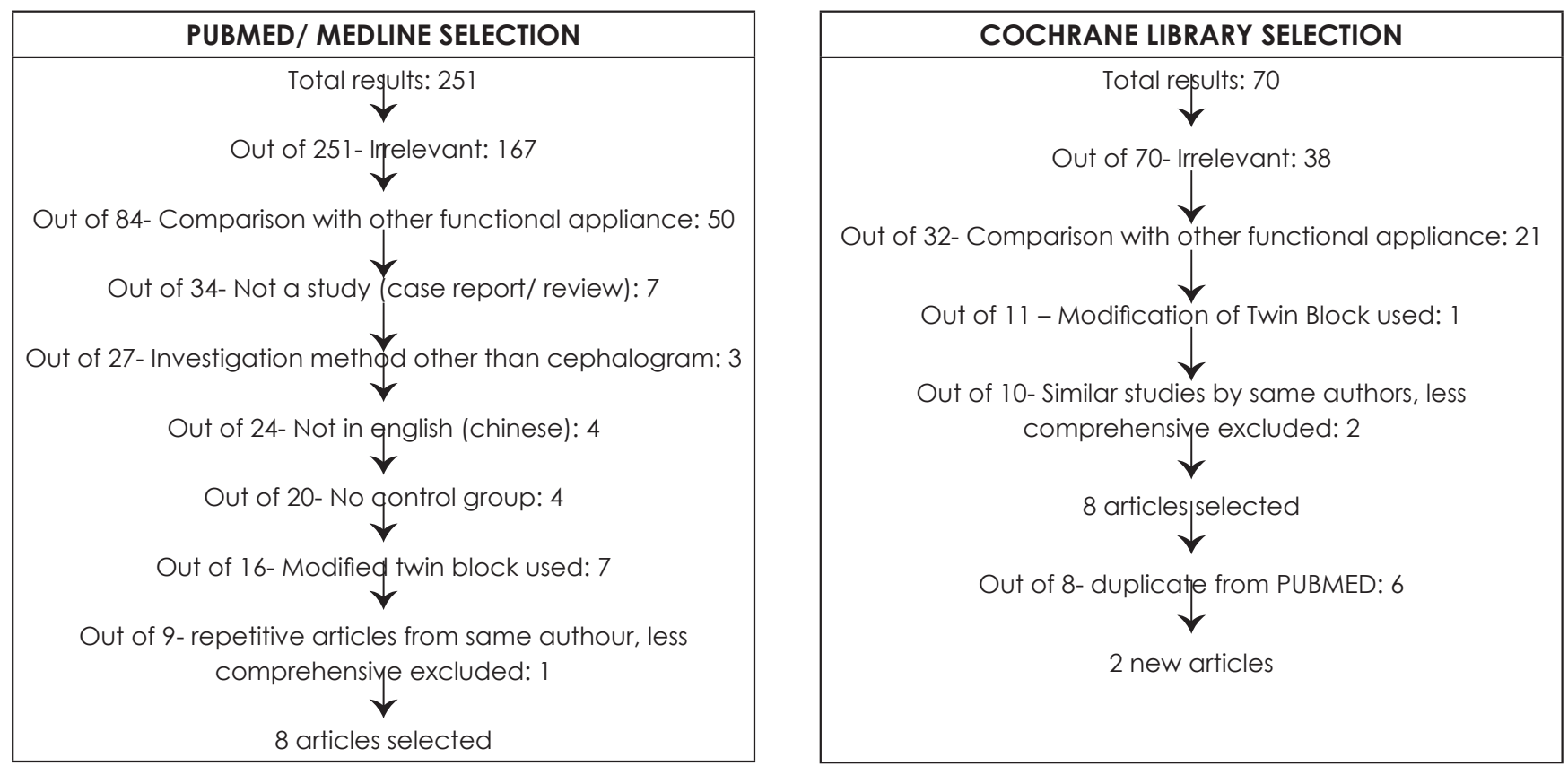

Flow Chart 2 


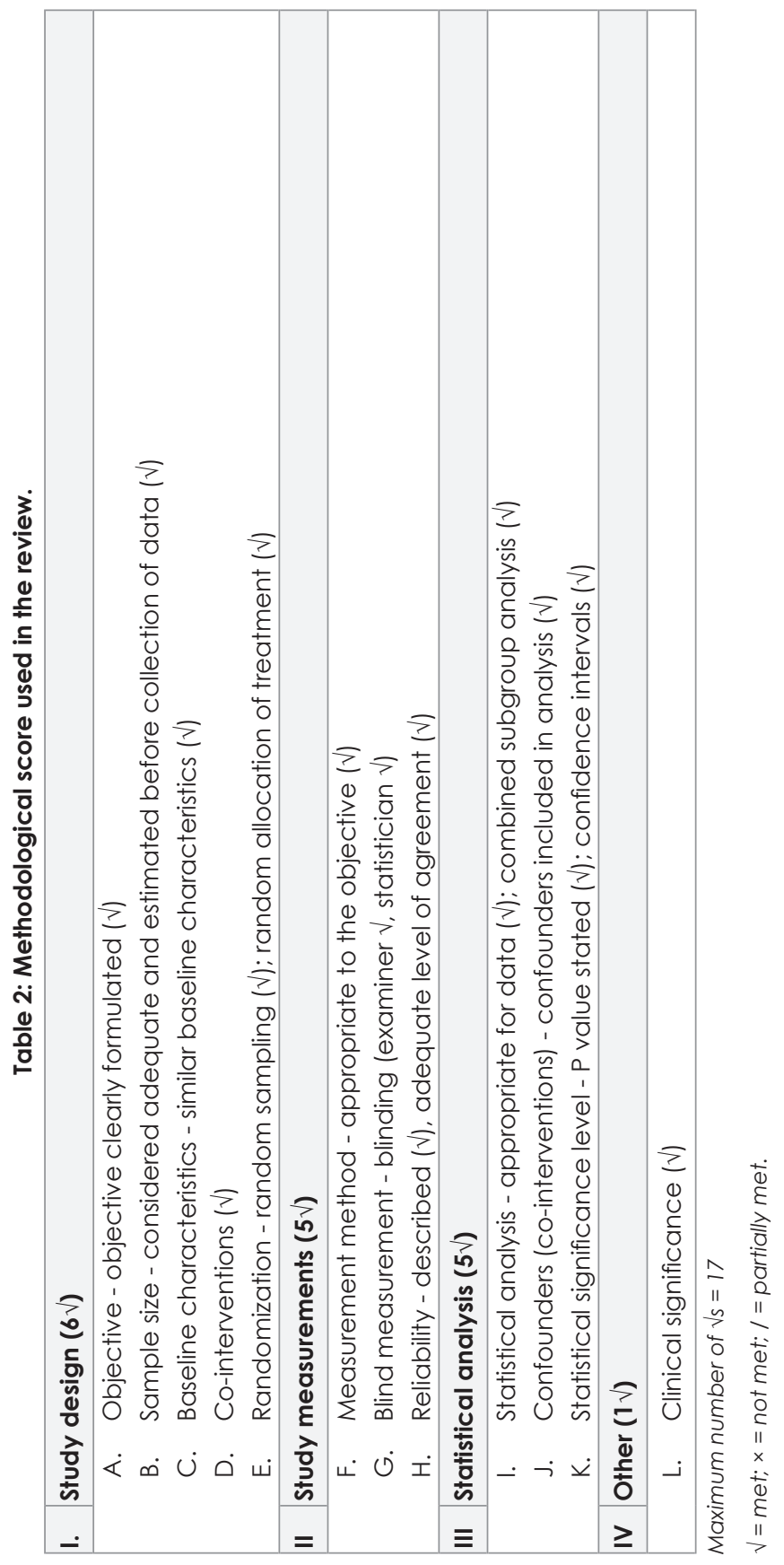

\begin{tabular}{|c|c|c|c|c|c|c|c|c|c|c|c|}
\hline & SD!̣ f૦ শs!y & - & $\Sigma$ & $\Sigma$ & \lrcorner & - & $I$ & $I$ & I & I & I \\
\hline & 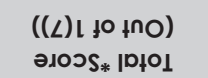 & \pm & $\simeq$ & $\simeq$ & \pm & $\underline{m}$ & $a$ & $=$ & 으 & 으 & $a$ \\
\hline & 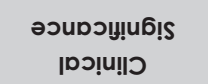 & $>$ & $>$ & $>$ & $>$ & $>$ & $>$ & $>$ & $>$ & $>$ & $>$ \\
\hline & 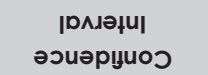 & $>$ & $>$ & $>$ & $>$ & $>$ & $>$ & $>$ & $>$ & $>$ & $>$ \\
\hline & әกןD^ d & $>$ & $>$ & $>$ & $>$ & $>$ & $>$ & $>$ & $>$ & $>$ & $>$ \\
\hline & $\begin{array}{c}\text { s!s/ןjpu } \\
\text { u! pəpn|כu| } \\
\text { sıəpunojuoJ }\end{array}$ & $>$ & $>$ & $>$ & $>$ & $>$ & $\times$ & $>$ & $>$ & $>$ & $>$ \\
\hline & 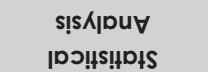 & $>$ & $>$ & $>$ & $>$ & $>$ & - & $>$ & $>$ & $>$ & $>$ \\
\hline 导 & 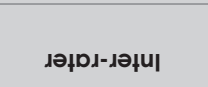 & $>$ & $>$ & $>$ & $>$ & $>$ & $\times$ & $\times$ & $x$ & $x$ & $\times$ \\
\hline$\stackrel{\overline{\mathbf{m}}}{\Xi}$ & ІәңDد-Dג & $>$ & $>$ & $>$ & $>$ & $>$ & $x$ & $>$ & $>$ & $>$ & $x$ \\
\hline & 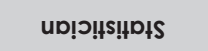 & $x$ & $x$ & $x$ & $x$ & $\times$ & $\times$ & $\times$ & $x$ & $x$ & $x$ \\
\hline$\overline{\text { Фे }}$ & 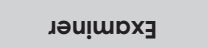 & $>$ & $x$ & $\times$ & $>$ & $\times$ & $\times$ & $\times$ & $\times$ & $x$ & $\times$ \\
\hline & $\begin{array}{c}\text { рочџәW } \\
\text { †นәuәлnsDəW }\end{array}$ & $>$ & $>$ & $>$ & $>$ & $>$ & $>$ & $>$ & $>$ & $>$ & $>$ \\
\hline$\frac{z}{\frac{2}{4}}$ & $\begin{array}{l}\text { uo!̣pวo॥I } \\
\text { uopupy }\end{array}$ & $>$ & $x$ & $\times$ & $>$ & $>$ & $\times$ & $\times$ & $\times$ & $\times$ & $\times$ \\
\hline 足 & $\begin{array}{l}\text { 6u!|dunss } \\
\text { mopupy }\end{array}$ & $\times$ & $\times$ & $x$ & $x$ & $x$ & $x$ & $x$ & $x$ & $x$ & $x$ \\
\hline & $\begin{array}{c}\text { suo!̣uәмдәңप!-०ว } \\
\text { оN }\end{array}$ & $>$ & $>$ & $>$ & $>$ & $>$ & $>$ & $>$ & $>$ & $>$ & $>$ \\
\hline & әu!|əsDg & $>$ & $>$ & $>$ & $>$ & $>$ & $>$ & $>$ & $\times$ & $x$ & $x$ \\
\hline & әz!s әdüs & - & - & $>$ & - & $>$ & - & $>$ & $>$ & $>$ & - \\
\hline & ә^!џગә!વ૦ & $>$ & $>$ & $>$ & $>$ & $>$ & $>$ & $>$ & $>$ & $>$ & $>$ \\
\hline & & 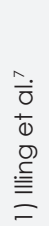 & 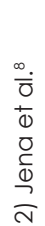 & $\begin{array}{l}\frac{a}{\bar{d}} \\
\overline{0} \\
\overline{0} \\
\overline{0} \\
\infty \\
0 \\
\overline{0} \\
\frac{1}{m}\end{array}$ & 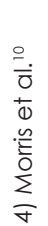 & 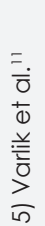 & $\begin{array}{l}\frac{0}{0} \\
\frac{1}{0} \\
2 \\
0 \\
0 \\
3 \\
0 \\
0 \\
0\end{array}$ & $\begin{array}{l}\frac{m}{\overline{0}} \\
\frac{1}{0} \\
\frac{0}{0} \\
\frac{\bar{v}}{\bar{v}} \\
\frac{\pi}{\pi}\end{array}$ & $\begin{array}{l} \pm \\
\overline{0} \\
\overline{0} \\
\bar{D} \\
\tilde{D} \\
\bar{D} \\
\bar{\infty}\end{array}$ & $\begin{array}{l}\frac{\varrho}{\overline{0}} \\
\bar{\Phi} \\
\overline{0} \\
\tilde{0} \\
0 \\
\stackrel{\infty}{a}\end{array}$ & 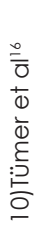 \\
\hline
\end{tabular}


Table 4: Summary of selected articles.

\begin{tabular}{|c|c|c|c|c|c|c|c|}
\hline & & & Sample Size & & & & \\
\hline & $\begin{array}{l}\text { Study } \\
\text { Type* }\end{array}$ & Total & Twin Block & Control & $\begin{array}{c}\text { Mean age of } \\
\text { combined groups at } \\
\mathrm{T} 1 \text { (years) }\end{array}$ & $\begin{array}{l}\text { Skeletal maturity } \\
\text { indicators }\end{array}$ & $\begin{array}{c}\text { Treatment } \\
\text { duration } \\
\text { (months) }\end{array}$ \\
\hline 1) Illing et al ${ }^{7}$ & $\mathrm{P}$ & 36 & 16 & 20 & 11.2 & - & 9 \\
\hline 2) Jena et $a l^{8}$ & $P$ & 35 & 25 & 10 & 11.4 & - & 12.78 \\
\hline 3) Lund and Sandler? & $P$ & 63 & 36 & 27 & 12.4 & - & 14.4 \\
\hline 4) Morris et al ${ }^{10}$ & $P$ & 36 & 16 & 20 & 11.2 & - & 9 \\
\hline 5) Varlik et al ${ }^{11}$ & $P$ & 50 & 25 & 25 & 11.9 & MP3 H stage & 8 \\
\hline 6) Dauvravu et al' ${ }^{12}$ & $P$ & 28 & 17 & 11 & $10-14$ years & $\begin{array}{l}\text { CVMI- III and IV } \\
\text { MP3-H stage }\end{array}$ & 11.5 \\
\hline 7) Khoja et $a^{13}$ & $P$ & 113 & 53 & 60 & 11.3 & CVMI- II, III, IV & $8-12$ \\
\hline 8) Baysal et all' & $P$ & 40 & 20 & 20 & 12.58 & MP3 S and H2 stage & 15.89 \\
\hline 9) Baysal et all ${ }^{15}$ & $P$ & 40 & 20 & 20 & 12.58 & MP3 S and H2 stage & 15.89 \\
\hline 10) Tümer et $\mathrm{al}^{16}$ & $P$ & 26 & 13 & 13 & 13 & - & $7-14$ \\
\hline
\end{tabular}

* $P=$ prospective, $R=$ retrospective; - =not mentioned

\section{Data items and collection}

Skeletal cephalometric findings denoting anteroposterior maxillary changes, mandibular changes, vertical changes, dental findings denoting effects on maxillary and mandibular molars and incisors, overjet and soft tissue findings such as the nasolabial angle, mentolabial sulcus and the position of upper and lower lip were collected.

\section{Risk of bias in individual studies}

Risk of bias was assessed through the evaluation of methodological quality study characteristics (Table 2). 5,17

Intra-rater reliability, interrater reliability, and blinding of examiner and/or statistician were considered. The studies were labeled as low risk, medium risk and high risk for bias. (Table 3).

\section{Summary measures}

Basic study characteristics for each of the 10 studies are mentioned in Table 4.

\section{RESULT}

\section{Selection and study characteristics}

A total of 10 articles meeting the inclusion criteria were selected as shown in Flow Chart 1 \& 2.

\section{Risk of Bias within Studies}

Out of the 10 studies 3 studies ${ }^{7,10,11}$ have a low risk of bias, 5 studies ${ }^{12-16}$ have a high risk of bias and remaining ${ }^{8,9}$ have a medium risk of bias.

\section{Antero-posterior effects on maxilla (Table 5)}

Illing et $a_{1},{ }^{7}$ Dauvravu et $a_{1},{ }^{12}$ Baysal et $a^{15}$ have reported a significant headgear effect. Whereas, Jena et $a_{1}{ }^{8}$ Lund and Sandler, ${ }^{9}$ Khoja et al, ${ }^{13}$ Baysal et al ${ }^{14}$ and Tümer et al $^{16}$ have reported maxillary restrictive effect which were statistically insignificant. Varlik et $\mathrm{al}^{11}$ reported no maxillary restrictive effect.

\section{Antero-posterior effects on mandible (Table 6)}

All authors have reported statistically significant increase in mandible.

\section{Vertical effects (Table 7)}

Illing et $\mathrm{al}^{7}{ }^{7}$ Lund and Sandler, ${ }^{9}$ Khoja et al, ${ }^{13}$ Baysal et $\mathrm{al}^{14}$ and Tümer et $\mathrm{al}^{16}$ have stated statistically significant increase in the vertical height and Baysal et $a^{15}$ have reported insignificant findings.

\section{Effects on maxillary molar (TABLE 8)}

Jena et al, ${ }^{8}$ Lund and Sandler, 9 Dauvravu et $\mathrm{al}^{12}$ and Tümer et $\mathrm{al}^{16}$ have revealed statistically significant restrictive effect on the maxillary molars and, Baysal et $\mathrm{al}^{14}$ have reported otherwise.

\section{Effect on mandibular molar (TABLE 9)}

Lund and Sandler, 9 Dauvravu et $\mathrm{al}^{12}$ and Tümer et $\mathrm{al}^{16}$ have reported statistically significant advancement of the mandibular molar. Baysal et $\mathrm{al}^{14}$ and Jena et $\mathrm{al}^{8}$ have reported statistically insignificant findings.

\section{Effect on maxillary incisors (TABLE 10)}

Except for Baysal et $\mathrm{al}^{14}$ and Baysal et $\mathrm{al}^{15}$ all other studies have reported statistically significant decrease in maxillary incisors inclination.

\section{Effect on mandibular incisors (TABLE 11)}

Except for Baysal et $\mathrm{al}^{14}$ and Baysal et $\mathrm{al}^{15}$ all 
Table 5: Skeletal antero-posterior effects on maxilla.

\begin{tabular}{|c|c|c|c|c|c|}
\hline \multirow{2}{*}{ Study } & \multirow{2}{*}{ Parameter } & \multicolumn{2}{|c|}{ Changes Reported } & \multirow{2}{*}{ P Value } & \multirow{2}{*}{ Significance S / NS } \\
\hline & & Control group & Treatment group & & \\
\hline 1) Illing et al. ${ }^{7}$ & SNA & 0.3 & -1.4 & $p<0.01$ & S \\
\hline 2) Jena et $\left.a\right|^{8}$ & - & 2.04 & -1.64 & $p=0.259$ & NS \\
\hline 3) Lund and Sandler9 & SNA & 0.3 & -0.1 & $p>0.05$ & NS \\
\hline 4) Varlik et $a^{11}$ & Point A to $Y$ axis(Se to ptm) & 0.2 & 1.2 & $p>0.05$ & NS \\
\hline 5) Dauvravu et al'2 & Ss/rlp (Pancherz analysis ) & - & -0.67 & $p=0.022$ & $S$ \\
\hline 6) Khoja et $a^{13}$ & SNA & $0.04 \pm 1.01$ & $-0.19 \pm 1.10$ & $p=0.168$ & NS \\
\hline 7) Baysal et al ${ }^{14}$ & Point A/olp (Pancherz analysis) & 1.35 & -0.45 & $p>0.05$ & NS \\
\hline 8) Baysal et all ${ }^{15}$ & SNA & 0.20 & -0.75 & $p=0.004$ & S \\
\hline 9) Tümer et all6 & SNA & 0.05 & -0.23 & $p>0.05$ & NS \\
\hline
\end{tabular}

$S=$ significant,$N S=$ not significant

Table 6: Skeletal antero-posterior effects on mandible.

\begin{tabular}{|c|c|c|c|c|c|}
\hline \multirow{2}{*}{ Study } & \multirow{2}{*}{ Parameter } & \multicolumn{2}{|c|}{ Changes Reported } & \multirow{2}{*}{ P Value } & \multirow{2}{*}{ Significance } \\
\hline & & Control group & Treatment group & & \\
\hline 1) Illing et al. ${ }^{7}$ & Ar to Gn & 1 & 3.2 & $(p<0.05)$ & $S$ \\
\hline 2) Jena et $\left.a\right|^{8}$ & - & $3.54 \mathrm{~mm}$ & $5.52 \mathrm{~mm}$ & $(p<0.01)$ & $S$ \\
\hline 3) Lund and Sandler? & SNB & 0.4 & 1.9 & $(p<0.001)$ & $S$ \\
\hline 4) Varlik et al ${ }^{11}$ & B to $Y$ axis(Se to ptm) & 0.4 & 4.2 & $(p<0.001)$ & $S$ \\
\hline 5) Dauvravu et al' ${ }^{12}$ & pg/ OLp (Pancherz analysis) & - & $4.88 \mathrm{~mm}$ & $(p=0.000)$ & $S$ \\
\hline 6) Khoja et al ${ }^{13}$ & SNB & $0.17 \pm 1.03$ & $1.73 \pm 1.22$ & $(p<0.001)$ & S \\
\hline 7) Baysal et al ${ }^{14}$ & pg/OLp (Pancherz analysis) & 2.12 & 4.62 & $(p=0.009)$ & S \\
\hline 8) Baysal et al15 & SNB & 0.45 & 2.07 & $(p<0.001)$ & $S$ \\
\hline 9) Tümer et $a^{16}$ & SNB & 0.31 & 1.77 & $(p<0.01)$ & $S$ \\
\hline
\end{tabular}

$S=$ significant, NS= not significant

Table 7: Vertical skeletal effects

\begin{tabular}{|c|c|c|c|c|c|}
\hline \multirow{2}{*}{ Study } & \multirow{2}{*}{ Parameter } & \multicolumn{2}{|c|}{ Changes Reported } & \multirow{2}{*}{ P Value } & \multirow{2}{*}{ Significance } \\
\hline & & Control group & Treatment group & & \\
\hline 1) Illing et al. ${ }^{7}$ & Lower anterior facial height & -0.3 & +2.7 & $(p<0.01)$ & $S$ \\
\hline 2) Lund and Sandler 9 & Total anterior facial height & 2.3 & 4.9 & $(p<0.001)$. & $S$ \\
\hline 3) Khoja et $a^{13}$ & Sn-GoGn & $-0.19 \pm 1.09$ & $0.60 \pm 2.45$ & $(p=0.029)$ & $S$ \\
\hline 4) Baysal et $\mathrm{al}^{14}$ & Lower anterior facial height & 2.05 & 3.85 & $(p=0.022)$ & $S$ \\
\hline 5) Baysal et all ${ }^{15}$ & Sn-GoGn & -0.62 & -0.25 & $(p>0.05)$. & NS \\
\hline 6) Tümer et $\mathrm{al}^{16}$ & Ar-Go-M & -0.73 & 2.31 & $(p<0.01)$ & $S$ \\
\hline
\end{tabular}

S= significant, NS= not significant

Table 8: Effects on maxillary molar

\begin{tabular}{|c|c|c|c|c|c|}
\hline \multirow{2}{*}{ Study } & \multirow{2}{*}{ Parameter } & \multicolumn{2}{|c|}{ Changes Reported } & \multirow{2}{*}{ P Value } & \multirow{2}{*}{ Significance } \\
\hline & & Control group & Treatment group & & \\
\hline 1) Jena et $a^{8}$ & - & $-0.36 \mathrm{~mm}$ & $-1.36 \mathrm{~mm}$ & $(p<0.05)$ & S \\
\hline 2) Lund and Sandler' & U6 -horizontal & 0.9 & -0.7 & $(p=0.05-0.01)$ & S \\
\hline 3) Dauvravu et al ${ }^{12}$ & (mi/RLp by Pancherz analysis) & - & $\begin{array}{c}-0.133 \pm \\
1.245 \mathrm{~mm}\end{array}$ & $(p=0.014)$ & S \\
\hline 4) Baysal et al ${ }^{14}$ & mi/OLp-pg/OLp (Pancherz analysis) & 0.27 & -0.52 & $(p>0.05)$ & NS \\
\hline 5) Tümer et $a^{16}$ & U6 to Ptv & 1.50 & -0.54 & $(p<0.01)$ & $S$ \\
\hline
\end{tabular}

S= significant, NS= not significant 
Table 9: Effects on mandibular molar

\begin{tabular}{|c|c|c|c|c|c|}
\hline \multirow{2}{*}{ Study } & \multirow{2}{*}{ Parameter } & \multicolumn{2}{|c|}{ Changes Reported } & \multirow{2}{*}{ P Value } & \multirow{2}{*}{ Significance } \\
\hline & & Control group & Treatment group & & \\
\hline 1) Jena et $\left.a\right|^{8}$ & - & $0.36 \mathrm{~mm}$ & $1.53 \mathrm{~mm}$ & $(p>0.05)$ & NS \\
\hline 2) Lund and Sandler9 & L6- horizontal & 1.0 & 4.7 & $(p=0.05-0.01)$ & $S$ \\
\hline 3) Dauvravu et al ${ }^{12}$ & $\begin{array}{l}\text { mi/RLp(d) minus pg/RLp(d) } \\
\text { (Pancherz analysis) }\end{array}$ & - & $0.666 \pm 1.496 \mathrm{~mm}$ & $(p<0.01)$ & $S$ \\
\hline 4) Baysal et al ${ }^{14}$ & mi/OLp-pg/OLp & -0.22 & 0.35 & $(p>0.05)$ & NS \\
\hline 5) Tümer et $\mathrm{al}^{16}$ & L6/PtV & 2.04 & 4.89 & $(p<0.01)$ & $S$ \\
\hline
\end{tabular}

$S=$ significant, NS= not significant

Table 10: Effects on maxillary incisor

\begin{tabular}{|c|c|c|c|c|c|}
\hline \multirow{2}{*}{ Study } & \multirow{2}{*}{ Parameter } & \multicolumn{2}{|c|}{ Changes Reported } & \multirow{2}{*}{ P Value } & \multirow{2}{*}{ Significance } \\
\hline & & Control group & Treatment group & & \\
\hline 1) Illing et $\mathrm{al}^{7}$ & U1 to maxillary plane & -1.9 & -9.1 & $(p<0.01)$ & $S$ \\
\hline 2) Jena et $a 1^{8}$ & - & $-0.53 \mathrm{~mm}$ & $-1.43 \mathrm{~mm}$ & $(p<0.001)$ & $S$ \\
\hline 3) Lund and Sandler' & U1 to maxillary plane & -0.2 & -11 & $(p<0.001)$ & $S$ \\
\hline 4) Morris et allo & - & - & - & - & - \\
\hline 5) Varlik et a ${ }^{11}$ & UI-SN & 0.2 & -2.6 & $(p<0.001)$ & $S$ \\
\hline 6) Dauvravu et al ${ }^{12}$ & is/RLp (Pancherz analysis) & - & $0.866 \pm 1.125 \mathrm{~mm}$ & $(p=0.054)$ & $S$ \\
\hline 7) Khoja et al ${ }^{13}$ & UI-SN & $1.12 \pm 4.19$ & $-4.66 \pm 5.44$ & $(p<0.001)$ & $S$ \\
\hline 8) Baysal et all'4 & is/OLp- A/OLp & 0.75 & 0.45 & $(p>0.05)$ & NS \\
\hline 9) Baysal et all ${ }^{15}$ & UI-SN & 1.62 & -1.47 & $(p>0.05)$ & NS \\
\hline 10) Tümer et $\mathrm{al}^{16}$ & U1/PtV & 1.58 & -1.50 & $(p<0.01)$ & $S$ \\
\hline
\end{tabular}

$S=$ significant, $N S=$ not significant

Table 11: Effects on mandibular incisor

\begin{tabular}{|c|c|c|c|c|c|}
\hline \multirow{2}{*}{ Study } & \multirow{2}{*}{ Parameter } & \multicolumn{2}{|c|}{ Changes Reported } & \multirow{2}{*}{ P Value } & \multirow{2}{*}{ Significance } \\
\hline & & Control group & Treatment group & & \\
\hline 1) Illing et $\mathrm{al}^{7}$ & Lower incisor to mandibular plane & -0.7 & +2 & $(p<0.001)$ & S \\
\hline 2) Jena et $\left.a\right|^{8}$ & - & $-0.59 \mathrm{~mm}$ & $+1.44 \mathrm{~mm}$ & $(p<0.001)$ & $S$ \\
\hline 3) Lund and Sandler? & Li- mandibular plane & 0.9 & 4.4 & $(p<0.001)$ & $S$ \\
\hline 4) Varlik et al"1 & IMPA & No change & 1.3 & $(p<0.001)$ & $S$ \\
\hline 5) Dauvravu et al ${ }^{12}$ & $\mathrm{li} / \mathrm{rlp}$ & & $1.40 \pm 1.638 \mathrm{~mm}$ & $(p=0.012)$ & S \\
\hline 6) Khoja et al ${ }^{13}$ & IMPA & $1.05 \pm 3.45$ & $4.30 \pm 3.91$ & $(p=0.001)$ & $S$ \\
\hline 7) Baysal et al'14 & li/olp-pg/olp & -0.40 & 0.15 & $(p>0.05)$ & NS \\
\hline 8) Baysal et all15 & IMPA & 0.57 & 0.92 & $(p>0.05)$ & NS \\
\hline 9) Tümer et al16 & Ll/ptv & 1.19 & 5.73 & $(p<0.01)$ & S \\
\hline
\end{tabular}

$S=$ significant, NS= not significant

other authors have reported statistically significant proclination of mandibular incisors.

\section{Effects on the Overjet (Table 12)}

All studies have reported a statistically significant decrease in the overjet.

\section{Soft tissue effects of Twin Block (Table 13)}

Morris et al, ${ }^{10}$ Varlik et al, ${ }^{11}$ Khoja et $a l^{13}$ and Baysal et al ${ }^{15}$ have reported statistically significant retrusive upper lip effect with a significant increase in nasolabial angle, whereas insignificant findings were reported by Morris et $\mathrm{al}^{10}$ and Baysal et $\mathrm{al}^{15}$ Morris et $\mathrm{al}^{10}{ }^{10}$ Khoja et $\mathrm{al}^{13}$ and Baysal et $\mathrm{al}^{15}$ have narrated a statistically significant lower lip protrusion, whereas, Varlik et $\mathrm{al}^{11}$ reported otherwise. Of these four studies, Morris et $\mathrm{al}^{10}$ revealed insignificant change whereas Varlik et $\mathrm{al}^{11}$ and Baysal et $a^{15}$ reported statistically significant increase in the mentolabial sulcus depth. 
Table 12: Effects on the overjet

\begin{tabular}{|c|c|c|c|c|c|}
\hline \multirow{2}{*}{ Study } & \multirow{2}{*}{ Parameter } & \multicolumn{2}{|c|}{ Changes Reported } & \multirow{2}{*}{ P Value } & \multirow{2}{*}{ Significance } \\
\hline & & Control group & Treatment group & & \\
\hline 1) Illing et al ${ }^{7}$ & Overjet & 0.8 & -5.7 & $(p<0.01)$ & $S$ \\
\hline 2) Jena et $\left.a\right|^{8}$ & Overjet & $0.37 \mathrm{~mm}$ & $-6.75 \mathrm{~mm}$ & $(p<0.001)$ & $S$ \\
\hline 3) Lund and Sandler? & Overjet & -0.3 & -7.9 & $(p<0.001)$ & $S$ \\
\hline 4) Dauvravu et al' ${ }^{12}$ & is/RLp-ii/RLp & & $-6.54 \pm 1.12 \mathrm{~mm}$ & $(p<0.000)$ & S \\
\hline 5) Khoja et al ${ }^{13}$ & Overjet & $-0.30 \pm 1.25$ & $-6.50 \pm 2.46$ & $(p<0.001)$ & S \\
\hline 6) Baysal et al ${ }^{14}$ & is/OLp-ii/OLp & 0.38 & -4.48 & $(p=0.000)$ & $S$ \\
\hline 7) Tümer et $a^{16}$ & Overjet & 0.27 & -7.46 & $(p<0.01)$ & $S$ \\
\hline
\end{tabular}

S= significant, NS= not significant

Table 13: Soft tissue effects

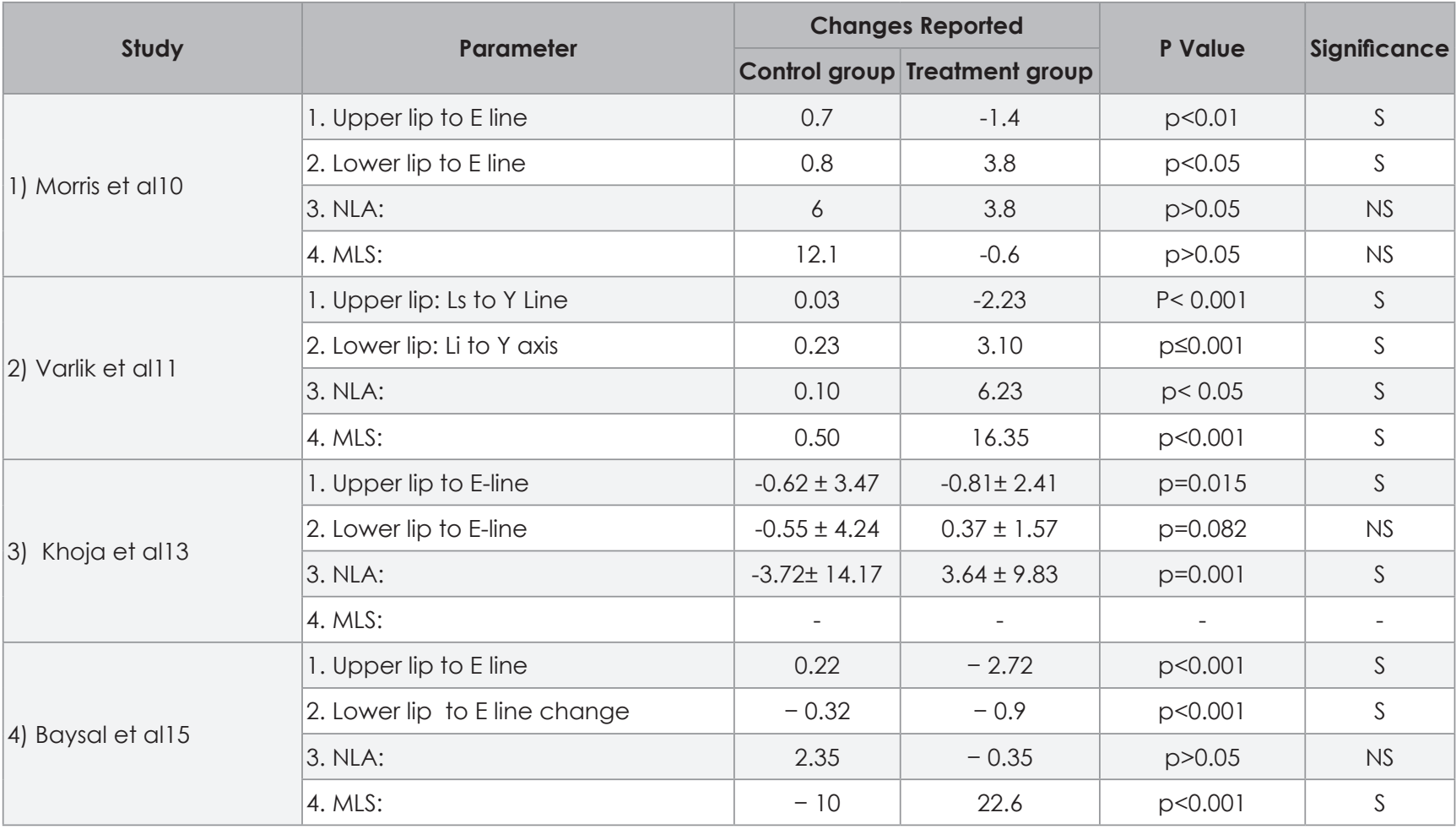

S= significant, NS= not significant

\section{DISCUSSION}

According to the results, restrictive maxillary effects of Twin block were reported by a few studies, $7,12,15$ whereas most studies reported no restrictive maxillary effect. $8,9,11,13,14,16$ All the studies reported a significant increase in the mandibular jaw base..$^{-16}$

Out of the 10 studies, only 6 studies have registered vertical skeletal changes. All have found statistically significant increase in vertical dimensions ${ }^{7,13-16}$ except for one study which gave insignificant results.?

Out of the studies that reported maxillary molar and mandibular molar changes only one study 14 reported insignificant changes while others showed a statistically significant maxillary molar restrictive effect and a mandibular molar advancement.8,9,12,16

Except for Two studies ${ }^{14,15}$ all other studies reported a significant decrease in the inclination of the maxillary incisors. ${ }^{7-9,11-13,16}$ Most of the studies reported a significant mesial mandibular incisor movement.8,9,11-13,16 Some studies have reported insignificant mandibular incisor change. ${ }^{7,14,15}$

Except for the 3 studies that did not report changes in the overjet, ${ }^{10,11,15}$ all have reported a significant decrease in the overjet. ${ }^{7-9,12-14,16}$ 
Studies reporting soft tissue parameters were only four. ${ }^{10,11,13,15}$ Equal number of studies found significant ${ }^{11,13}$ and insignificant ${ }^{10,15}$ changes in the Nasolabial angle. Two studies found significant ${ }^{11,15}$ change in the Mentolabial sulcus whereas one study found an insignificant result. ${ }^{10}$ All studies found a significant posterior positioning of the Upper lip, 10,11,13,15 and anterior positioning of the Lower lip position, $10,11,15$ except for one. ${ }^{13}$

\section{CONCLUSION}

This systematic review has aimed to find out the dental, skeletal and soft tissue effects of the conventional Twin Block appliance as compared with the control. From this systematic review it can be safely concluded that the conventional Twin Block appliance is indicated for individuals diagnosed with a Class II malocclusion with a horizontal growth pattern and a frank mandibular retrognathism. The use of this appliance is contraindicated in the cases of maxillary prognathism. Effective and favourable soft tissue changes are seen. However, some inevitable dental changes mainly lower anterior proclination are strongly reported which need to be timely addressed.

\section{REFERENCES}

1. Clark W, Clark WJ. Twin block functional therapy. JP Medical Ltd; 2014 Sep 30.

2. Trenouth MJ. A functional appliance system for the correction of Class II relationships. British journal of orthodontics. 1989 Aug; 16 (3):16976.

3. Proffit WR, Fields Jr HW, Sarver DM. Contemporary orthodontics. Elsevier Health Sciences; 2006 Dec 8.

4. Flores-Mir C, Major PW. Cephalometric facial soft tissue changes with the Twin block appliance in Class II division 1 malocclusion patients: a systematic review. The Angle Orthodontist. 2006 Sep;76(5):876-81.

5. Ehsani S, Nebbe B, Normando D, Lagravere MO, Flores-Mir C. Short-term treatment effects produced by the Twin-block appliance: a systematic review and meta-analysis. European Journal of Orthodontics. 2015 Apr 1;37(2):170-6.

6. Cozza P, Baccetti T, Franchi L, De Toffol L, McNamara Jr JA. Mandibular changes produced by functional appliances in Class II malocclusion: a systematic review. American Journal of Orthodontics and Dentofacial Orthopedics. 2006 May 1;129(5):599-e1.

7. Illing HM, Morris DO, Lee RT. A prospective evaluation of Bass, Bionator and Twin Block appliances. Part I-The hard tissues. The European Journal of Orthodontics. 1998 Oct 1;20(5):501-16.

8. Jena AK, Duggal R, Parkash H. Orthopedic and orthodontic effects of twin-block appliance. Journal of Clinical Pediatric Dentistry. 2005 Apr 1;29(3):225-30.

9. Lund DI, Sandler PJ. The effects of Twin Blocks: a prospective controlled study. American Journal of Orthodontics and Dentofacial Orthopedics. 1998 Jan 1;113(1):104-10.

10. Morris DO, Illing HM, Lee RT. A prospective evaluation of Bass, Bionator and Twin Block appliances. The European Journal of Orthodontics. 1998 Dec 1;20(6):663-84.

11. Varlık SK, Gültan A, Tümer N. Comparison of the effects of Twin Block and activator treatment on the soft tissue profile. The European Journal of Orthodontics. 2008 Apr 1;30(2):128-34.

12. Dauravu LM, Vannala $\vee$, Arafath M, Singaraju GS, Cherukuri SA, Mathew A. The assessment of sagittal changes with twin block appliance in patients with decelerating growth phase. Journal of clinical and diagnostic research: JCDR. 2014 Dec;8(12):ZC81.

13. Khoja A, Fida M, Shaikh A. Cephalometric evaluation of the effects of the Twin Block appliance in subjects with Class II, Division 1 malocclusion amongst different cervical vertebral maturation stages. Dental press journal of orthodontics. 2016 Jun;21 (3):73-84.

14. Baysal A, Uysal T. Dentoskeletal effects of Twin Block and Herbst appliances in patients with Class II division 1 mandibular retrognathy. European journal of orthodontics. 2014 Apr 1;36(2):164-72.

15. Baysal A, Uysal T. Soft tissue effects of Twin Block and Herbst appliances in patients with Class II division 1 mandibular retrognathy. The European Journal of Orthodontics. $2013 \mathrm{Feb}$ 1;35(1):71-81.

16. Tümer N, Gültan AS. Comparison of the effects of monoblock and twin-block appliances on the skeletal and dentoalveolar structures. American journal of orthodontics and dentofacial orthopedics. 1999 Oct 1;116(4):460-8.

17. Lagravere MO, Major PW, Flores-Mir C. Long-term skeletal changes with rapid maxillary expansion: a systematic review. The Angle Orthodontist. 2005 Nov;75(6):1046-52. 